\title{
Etnobotánica, anatomía y caracterización físico-química del aceite esencial de Baccharis obovata Hook. et Arn. (Asteraceae: Astereae)
}

\author{
Soledad Molares ${ }^{1}$, Silvia Beatriz González ${ }^{2}$, Ana Ladio ${ }^{1,4}$ y María Agueda Castro ${ }^{3}$
}

Recibido: 14/07/2008. Aceptada: 29/10/2008

\begin{abstract}
RESUMEN - (Etnobotánica, anatomía y caracterización físico-química del aceite esencial de Baccharis obovata Hook. et Arn. (Asteraceae: Astereae)). Baccharis obovata Hook. et Arn. es una especie aromática conocida como "wautro", y forma parte destacada de la herbolaria Mapuche de la Patagonia Argentina. Los objetivos fueron investigar la etnobotánica, la anatomía del vástago, y caracterizar físicoquímicamente el aceite esencial presente en el mismo. Se realizaron entrevistas a pobladores de dos comunidades Mapuches del noroeste de la Patagonia Argentina, y se colectó material vegetal para su observación al microscopio óptico y de barrido ambiental, como así también, para la hidrodestilación del aceite esencial. Los informantes indicaron que la planta es utilizada en forma consensuada para el tratamiento de alopecia, caspa, diversas dermatitis, y cefaleas. Las principales características de la hoja son: mesofilo isobilateral, estomas anomocíticos, tricomas glandulares pluricelulares, y cavidades esquizógenas secretoras de aceites esenciales. El tallo primario presenta tricomas y cavidades secretoras semejantes a los de las hojas, colénquima en las costillas, clorénquima en los valles interrumpido por espacios de aire, cilindro vascular continuo, endodermis uniseriada y por sectores biseriada, esclerénquima discontinuo, y cristales aciculares en células periféricas de médula. Las determinaciones físico-químicas que se aportan son rendimiento medio anual, tiempo óptimo de destilación, densidad relativa, rotación específica e índice de refracción. B. obovata es una especie cosmecéutica de interés, este trabajo contribuye a su conocimiento general, aportando información para futuras tareas de control de calidad y para su conservación biocultural.
\end{abstract}

Palabras claves: aceites esenciales, anatomía foliar y caulinar, Baccharis obovata Hook. et Arn., Mapuche, usos medicinales

\begin{abstract}
Ethnobotany, anatomy and physicochemical characterization of essential oil of Baccharis obovata Hook. et Arn. (Asteraceae: Astereae)). Baccharis obovata Hook. et Arn. is an aromatic species known as "wautro", and is an important part of the Mapuche herbolarium of Patagonia Argentina. The objectives were to investigate its ethnobotany and shoot anatomy, as well as to characterize physically and chemically its essential oil. Rural informant-interviews were conducted in two Mapuche communities of Northwestern Patagonia, Argentina. In addition, we collected plant material for observation at optical and scanning environmental microscope, and for hydro-distillation of essential oil. Informants cited with a high level of consensus that this plant is principally used for hair loss, dandruff, some dermatitis, and headaches. The main leaf features were: isobilateral mesophyll, anomocytic stomata, glandular and pluricellular trichomas and squizogen cavities that secrete essential oils. Primary stem presented trichomas and secretory cavities similar to those of the leaves, collenchyma in ribs, chlorenchyma in valleys interrupted by air spaces, continuous vascular cylinder, uni-seriated and by sectors bi-seriated endodermis, discontinuous sclerenchyma, and acicular crystals in peripheral marrow cells. In terms of its essential oil, annual average yield, optimum time for distillation, relative density, specific rotation and refractive index was also obtained. B. obovata is an interesting cosmeceutical species, this work contributes to its general knowledge providing useful information for future quality control tasks, in addition to its bio-cultural conservation.
\end{abstract}

Keywords: Baccharis obovata Hook. et Arn., essential oils, Mapuche, medicinal uses, leaf and stem anatomy

\section{Introducción}

Baccharis L. (Asteraceae: Astereae) es un género con una riqueza aproximada de 400 especies. Su distribución geográfica es exclusivamente americana, extendiéndose desde el sur de los Estados Unidos hasta el extremo austral de Argentina y Chile. En esta vasta área se encuentra profusamente diversificado, ocupando gran variedad de ambientes y constituyendo un importante elemento en numerosas formaciones vegetales. En la República Argentina está representado por 96 especies (Giuliano \& Ariza Espinar 1999), 23 de estas creciendo en la Patagonia (Cabrera 1971).

Tradicionalmente, estas especies han formado parte importante de las farmacopeas de todo el país (Ariza Espinar 1969; Cortadi et al. 1999; Feresin et al. 2003).

\footnotetext{
1 Laboratorio Ecotono, INIBIOMA-CONICET, Quintral 1250, Bariloche (8400), Río Negro, Argentina

2 Universidad Nacional de la Patagonia San Juan Bosco, Facultad de Ciencias Naturales, Laboratorio Química Orgánica, Ruta 259, Esquel (9203), Chubut, Argentina

3 Universidad Nacional de Buenos Aires, Facultad Ciencias Exactas y Naturales, Laboratorio Anatomía vegetal, Ciudad Universitaria 1428, Buenos Aires, Argentina

4 Autor para correspondencia: aladio@crub.uncoma.edu.ar
} 
En la Patagonia en particular, se conocen y usan aproximadamente 10 especies, y su aprovechamiento se registra tanto en comunidades rurales como suburbanas (p.e.: Martínez Crovetto 1980; Conticello et al. 1997; Kutschker et al. 2002; González \& Molares 2004; Estomba et al. 2006; Ladio 2006). Son ejemplos las "carquejas" de tallos alados (B. trimera (Less.) DC., B. crispa Spreng., B. articulata (Lam.) Pers. y $B$. sagittalis (Less.) DC.), que preparadas en infusiones, se emplean como colagogas (Ariza Espinar 1969); y la "chilca" (B. salicifolia (Ruiz et Pav.) Pers.), usada en la elaboración de yesos para fracturas, como vermífuga, anti-inflamatoria, antitumoral y anti-ulcerosa (Martínez Crovetto 1980; Ladio 2006).

El uso medicinal extendido de este género, muy probablemente se encuentre vinculado a la presencia y actividad biológica de flavonoides y otros compuestos fenólicos, cumarinas y/o aceites esenciales que normalmente suelen poseer (Budel et al. 2003; Abad \& Bermejo 2007). Los estudios farmacológicos realizados sobre parte de los componentes químicos, o bien sobre los exudados completos, han demostrado principalmente, actividad anti-inflamatoria, antioxidante, anti-microbiana y anti-fúngica, además de las digestivas, hepáticas y renales más conocidas (Feresin et al. 2003; Abad \& Bermejo 2007). En cuanto a los aceites esenciales aislados y descriptos, son numerosos los trabajos realizados sobre especies argentinas (p.e.: Zunino et al. 1997; 2000; 2004; Cobos et al. 2001; Malizia et al. 2005a).

Baccharis obovata Hook. et Arn. por su parte, es una especie aromática de importancia etnobotánica para las poblaciones Mapuches de la Patagonia argentinochilena. Es una especie medicinal con cualidades terapéuticas, como las analgésicas, dermatológicas y atenuantes del frío, además de la más popularizada, relacionada con el control de la caída del cabello (Martínez Crovetto 1980; Kutschker et al. 2002).

Los estudios químicos realizados sobre sus partes aéreas, revelan la presencia de aceites esenciales, y detectan como principales componentes el $\alpha$-tuyeno, $\alpha$-pineno, $\beta$-pineno, sabineno, mirceno, limoneno, $\alpha$-terpineno, $\gamma$-terpineno y terpin-4-ol (Malizia et al. 2005 b). Sin embargo, y siendo una especie potencialmente útil para la atención primaria de la salud, debe mencionarse que aún se desconoce información etnobotánica, anatómica de los órganos empleados y físico-química del aceite esencial. Esta información resulta fundamental para su eventual validación científica y control de calidad (Chaudhury 1992; Cortadi et al. 1999).

Otros parámetros de importancia también por estudiar y conectar entre sí, son las propiedades organolépticas de la planta y los aceites esenciales (WHO 1998; Bandoni 2003). Desde la perspectiva etnobotánica, es posible dicho estudio dentro de un contexto simbólicocultural. Las interpretaciones culturales de las diferentes propiedades organolépticas pueden ser vinculadas a la existencia de aceites u otros componentes químicos por medio de técnicas de laboratorio (Messer 1991). Del mismo modo, se pueden relacionar las características organolépticas percibidas por la población estudiada, con determinados usos, formas de preparación y administración propios de la medicina local (Leonti et al. 2002). Las percepciones registradas en laboratorio permiten por su parte, establecer estándares de calidad y seguridad (Bandoni 2003).

Desde una aproximación integrativa que une lo etnobotánico, lo anatómico y lo físico-químico, los objetivos planteados fueron: 1) indagar sobre el conocimiento etnobotánico actual en dos comunidades Mapuches del noroeste de la Patagonia, con énfasis en sus aplicaciones medicinales, 2) medir la importancia cultural de la especie y sus diferentes usos 3) describir y detectar percepciones organolépticas vinculadas con su reconocimiento y uso, 4) identificar las estructuras secretoras de producción y liberación de los aceites esenciales, 5) obtener parámetros micrográficos de los órganos empleados, 6) determinar características físicoquímicas del aceite esencial, tales como rendimiento medio anual en peso oreado y seco, tiempo óptimo de destilación, densidad relativa, rotación específica e índice de refracción, y 7) describir características organolépticas del aceite esencial en términos estandarizados.

\section{Material y métodos}

Sitio de estudio - La indagación etnobotánica se realizó en las comunidades de Lago Rosario (456 habitantes) ( $43^{\circ} 15^{\prime} \mathrm{S}$ y $\left.71^{\circ} 21^{\prime} \mathrm{O}\right)$ y de Nahuelpan (60 habitantes) ( $42^{\circ} 57^{\prime} \mathrm{S}$ y $\left.71^{\circ} 10^{\prime} \mathrm{O}\right)$, en el Noroeste de la provincia de Chubut, Patagonia Argentina. Ambas comunidades se encuentran distantes entre sí a no más de $50 \mathrm{~km}$, y se conforman principalmente de Mapuches y en menor medida criollos (Instituto Nacional De Estadística y Censos 2001). La lengua nativa es el Mapuzungun ("lengua de la tierra"), pero en la actualidad sólo unos pocos la hablan activamente, y son generalmente ancianos bilingües. Su principal actividad económica es la ganadería, con la cría de ovinos y caprinos para la producción de lana y carne, y en menor medida la venta de artesanías al turismo nacional e internacional. Los centros poblacionales más importantes y cercanos son Esquel $(10 \mathrm{~km}$ desde Nahuelpan, $40 \mathrm{~km}$ desde Lago Rosario) y Trevelin (30 km desde Nahuelpan, $20 \mathrm{~km}$ desde Lago Rosario), donde se encuentran los principales comercios, centros oficiales de salud, comunicación y educación. 
580 Molares, González, Ladio \& Castro: Etnobotánica, anatomía y caracterización físico-química del aceite esencial...

Los ambientes naturales donde se encuentran inmersas las comunidades presentan diferentes grados de perturbación antrópica, producto principalmente del sobrepastoreo, la deforestación del bosque nativo y los incendios forestales, lo que se encuentra relacionado en parte, a la escasa superficie de tierra comunal, a la falta de oportunidades laborales en la sociedad, a procesos de emigración y de deagrarización (Catalán Labarías \& Ramos Antiqueo 2008).

Las características climáticas generales de la región someten a ambas poblaciones a rigurosas condiciones de adversidad. Incluyen fuertes vientos y heladas durante todo el año, intensas nevadas en invierno, y estación seca en verano. La vegetación se distribuye de acuerdo a un gradiente de precipitaciones que va desde los $400 \mathrm{~mm}$ anuales al oriente, hasta los $2000 \mathrm{~mm}$ anuales al occidente, como así también, a la geomorfología y edafología variables de la pre-cordillera andina (Dimitri 1977).

Fitogeográficamente, Lago Rosario se ubica en una zona de transición entre el distrito del Bosque caducifolio, provincia Subantártica y el distrito Patagónico Occidental, provincia Patagónica (Cabrera 1971). Las características generales son las particulares del ecotono bosque-estepa patagónico, con precipitaciones que rondan los 700-800 $\mathrm{mm}$ anuales. Las especies características son las arbóreas Nothofagus antarctica (G. Forst.) Oerst., Lomatia hirsuta (Lam.) Diels ex J.F. Macbr., Schinus patagonicus (Phil.) I.M. Johnst. ex Cabrera y Austrocedrus chilensis (D. Don) Pic.Serm. et Bizzarri, y en las zonas más altas Nothofagus pumilio (Poepp. et Endl.) Krasser.; las arbustivas Berberis spp., Mulinum spinosum (Cav.) Pers. y Senecio spp., entre otras; y las herbáceas Geranium spp., Mutisia spp., Fragaria chiloensis (L.) Duchense f., Poa spp., Bromus spp., Solidago chilensis Meyen y Urtica magellanica Poir., entre otras.

Nahuelpan por su parte, se ubica en el extremo oeste del distrito Patagónico Occidental, provincia Patagónica (Correa 1998), con precipitaciones que rondan los 400$500 \mathrm{~mm}$ anuales. La vegetación es la típica de la estepa gramino-arbustiva patagónica, con la presencia de gramíneas de los géneros Stipa spp., Festuca spp. y Cortadeira sp.; subarbustos de Mulinum spinosum, y arbustos dispersos de Berberis spp. y Adesmia volckmannii Phil.; en los alrededores de los mallines y ojos de agua crecen Juncus spp., Schinus patagonicus y Discaria spp., entre otras.

Etnobotánica - Este trabajo fue realizado entre los meses de diciembre y marzo de los años 2005 y 2006, y entre octubre y diciembre de 2007, y es parte de una investigación mayor sobre la flora medicinal aromática del noroeste de la Patagonia Argentina.

A un total de 30 pobladores de Lago Rosario y 15 de Nahuelpan (promedio de edad $=59 \pm 15$ años), se les solicitó que enlistaran las especies medicinales que conocen y/o usan (Alexiades 1996), a fin de poder determinar si B. obovata surgía espontáneamente en las listas, y en qué orden de aparición lo hacía. Otras variables indagadas fueron: nombres vulgares de la especie, usos medicinales, partes de la planta empleada, formas de preparación y administración, posibles combinaciones con otras especies, y las características organolépticas (aroma, sabor y aspecto) percibidas y relacionadas con su reconocimiento, selección y uso.

Para el análisis de la importancia cultural de B. obovata se emplearon distintas medidas de cuantificación: 1) porcentaje de citas $=\left(f n / \mathrm{N}_{1}\right) \times 100$, donde $f n$ es la frecuencia absoluta de B. obovata y $\mathrm{N}_{1}$ el número total de entrevistados $\left(\mathrm{N}_{1}=45\right)$ (Ladio 2006); 2) ranking promedio $=" p n / \mathrm{N}_{2}$, donde $p n$ es la posición u orden de aparición de $B$. obovata en el enlistado del entrevistado $n$ y $\mathrm{N}_{2}$ es el número total de entrevistados que mencionan a B. obovata (Alexiades 1996); 3) valor de uso total= UVs= "UVis $/ \mathrm{N}_{2}$, donde $\mathrm{UV}$ is es el número promedio de usos $i$ mencionados por cada entrevistado (Phillips 1993); y 4) consenso del uso $i=$ (número de entrevistados que informan sobre el uso $\left.i / \mathrm{N}_{2}\right) \times 100$ (Alexiades 1996; Ladio 2006).

Anatomía - Se trabajó con hojas adultas y tallos primarios en estado fresco de 30 ejemplares de la especie, 10 colectados en las inmediaciones de la comunidad de Lago Rosario y 20 en la de Nahuelpan. Se realizaron cortes transversales con micrótomo de deslizamiento y rotativo tipo Minot, por la porción media de la lámina, en el caso de las hojas, y entre el nudo 11 y el 13, en el caso de los tallos (el diámetro promedio de estos fue de 1387,5 $\mu \mathrm{m}$ $\pm 85,4 \mu \mathrm{m})$. Las coloraciones se realizaron con safranina y azul de metileno (D'Ambrogio de Argüeso 1986). Los aceites esenciales se localizaron utilizando Sudán IV (Cutter 1978; D'Ambrogio de Argüeso 1986; WHO 1998). Cabe mencionar que cuando este reactivo da positivo en sustancias asociadas a estructuras secretoras como las cavidades esquizógenas descriptas, se puede suponer con un margen de error muy bajo, que las sustancias reaccionantes son aceites esenciales (Fahn 1979; Duke 1994).

El indumento fue observado en trozos de epidermis obtenidos según el método de $\mathrm{KOH}$ al $3 \%$ y luego coloreados con safranina diluida (D'Ambrogio de Argüeso 1986).

Las dimensiones que se aportan son el promedio de treinta mediciones como mínimo.

Todas las observaciones se realizaron con un microscopio óptico Leitz Biomed, y se documentaron mediante foto-micrografías digitales con una cámara JVC GC-Q x5 HD. 
Complementariamente, y a fin de visualizar con mayor claridad la presencia de cristales, células secretoras y cutícula, se realizaron observaciones ultraestructurales con microscopio electrónico de barrido ambiental (MEBA), para lo cual se dispusieron trozos del material sin previo tratamiento, directamente sobre la platina de un microscopio Philips XL30 adosado con microsonda dispersiva de energía para análisis, aplicando una aceleración de voltaje de 20,0 kV.

Fitoquímica-El aceite esencial de hojas y tallos primarios fue extraído mediante hidrodestilación con un aparato tipo Clevenger similar al descrito por la Farmacopea Europea (1988), previa aireación a temperatura ambiente y a la sombra, por un lapso de 48 horas. Se destilaron 12 muestras de material vegetal colectado en inmediaciones de la comunidad de Nahuelpan (cada una compuesta por material perteneciente a cinco-seis individuos), las cuales fueron extraídas mensualmente durante el período abril 2001 - abril 2002.

Las determinaciones físico-químicas realizadas fueron: rendimiento medio anual en peso oreado y seco, tiempo óptimo de destilación, densidad relativa, rotación específica e índice de refracción. Las mismas fueron seleccionadas por lo útiles que resultan en la caracterización de los aceites esenciales, como así también, en la detección de adulteraciones y/o envejecimientos (Bandoni 2003). Las medidas que se aportan son el resultado del promedio de los valores calculados para cada mes. El análisis de la variación anual cuali-cuantitativa del aceite esencial será presentado en una próxima contribución.

El rendimiento se calculó mediante la relación: $\mathrm{mL}$ de aceite esencial $/ \mathrm{kg}$ de material vegetal oreado. Para expresar los rendimientos en $\mathrm{Kg}$ de material vegetal seco, se calculó un factor de corrección: F.c. $=100 / 100 * \%$ humedad. El \% de humedad se determinó con la fórmula: (Pi - Pf/Pi)*100, donde Pi es el peso inicial, y Pf es el peso final.

El tiempo óptimo de destilación se determinó construyendo una curva de acumulación, para la cual se ingresaron los valores del volumen de aceite esencial obtenido a cada hora del proceso de destilación, a partir de la hora de inicio.

La densidad relativa $\delta^{20}{ }^{\circ} \mathrm{C}$ se calculó utilizando un picnómetro de vidrio de $2 \mathrm{~mL}$ y siguiendo los pasos establecidos por las normas del Instituto Argentino de Normalización y Certificación- Sociedad Argentina para la Investigación de Productos Aromáticos (IRAMSAIPA) $N^{\circ} 185-04$.

La rotación específica $[\alpha]$ se calculó con un polarímetro SR-6 PolyScience, con luz de sodio y se siguieron los pasos establecidos por las normas IRAM-SAIPA $\mathrm{N}^{\circ}$ 185-07. En este caso se emplearon muestras del aceite diluidas en ciclohexano.

El índice de refracción se obtuvo con un refractómetro de Abbe- 3L Spectronic con luz de sodio y se siguieron las normas IRAM-SAIPA $\mathrm{N}^{\circ}$ 185-05. Cuando las determinaciones no se realizaron a la temperatura de referencia (20 ÚC), se aplicó la siguiente fórmula de corrección: $\mathrm{n}^{20^{\circ} \mathrm{C}}=\mathrm{n}^{\mathrm{t}^{\mathrm{t}}}+0,0004\left(\mathrm{t}^{\prime}-\mathrm{t}\right)$. Siendo $\mathrm{n}^{20}{ }^{\circ} \mathrm{C}$ el índice de refracción del aceite esencial a $20^{\circ} \mathrm{C}$, $\mathrm{n}^{\mathrm{t}}=$ el valor leído a la escala del aparato a la temperatura t', 0,0004= el valor promedio de la corrección en grados Celsius, aplicable a la mayoría de los aceites esenciales, t'= la temperatura a la cual se efectuó la determinación en grados Celsius y $\mathrm{t}=20^{\circ} \mathrm{C}$.

Las características organolépticas analizadas en el laboratorio fueron: olor, color y apariencia. Para la valoración olfativa se siguió, en líneas generales, el procedimiento propuesto por las normas IRAM-SAIPA $\mathrm{N}^{\mathrm{o}}$ 186-01, indicándose: 1) Nota inicial o nota de salida (primera sensación olfatoria de corta duración, experimentada al oler una banda de papel recientemente impregnada con el aceite esencial), 2) Nota media o nota de cuerpo (principal sensación experimentada al oler la banda de papel, después de ser expuesta a la atmósfera durante algún tiempo), 3) Nota residual o nota de fondo (sensación experimentada al oler la banda de papel, después del tiempo suficiente para que la nota inicial y la media hayan desaparecido), 4) Nota secundaria (olor extraño adicional al normal, experimentado durante alguna etapa de la evaporación). Las diferentes notas se describieron utilizando categorías relacionadas con lo vegetal, las que a su vez, se calificaron con diferentes adjetivos cuando se consideró necesario hacerlo. También se anotaron la Fuerza (intensidad de la impresión olfatoria) y la Persistencia (duración del aroma en una banda de papel impregnada por este).

Material depositado: B. obovata Hook. et Arn: ARGENTINA. Chubut: Lago Rosario, V/2006, fl. fr., Molares (BCRU).

\section{Resultados}

Etnobotánica - Los índices cuantitativos indican que B. obovata tiene un uso prevalente y consensuado en la zona de estudio. El $47 \%$ de los informantes (22 personas) menciona durante los enlistados libres que conoce y usa o usó durante la infancia a B. obovata ("wautro"). El ranking de la especie es intermedio, es decir su posición promedio en las listas, es la 16 , siendo 26 el número medio de especies nombrado por persona. El valor de uso total (UVs) es 1,4 es decir que en términos generales las personas la utilizan para pocas (menos de dos) dolencias distintas. El 47,6\% de las personas indica su uso para la limpieza del cabello y cuero cabelludo, 
sirviendo además como fijador de los peinados (trenzas y otros recojidos); el 23,8\% para la alopecia; el 23,8\% para el control de la caspa; el 19\% para tratar el acné y distintas dermatitis en la cara, como la "zarnilla" contagiada por el ganado ovino (ácaros de la sarna ovina en Patagonia: ej. Sarcoptes scabiei), el 14,3\% lo usa como analgésico del dolor de cabeza, reuma o dolor de huesos; el $4,8 \%$ para problemas respiratorios y el $4,8 \%$ para combatir la pediculosis.

En todos los casos, salvo para el tratamiento de problemas respiratorios en que se inhala el vapor surgido de la decocción de las partes aéreas, el uso es externo. La preparación consiste en hacer una decocción de las ramas frescas o secas, para luego emplearla en lavajes de la zona afectada, sin posterior enjuague. En el uso contra las dermatitis, la decocción puede combinarse con Erodium cicutarium (L.) L'Hér. ex Aiton (“alfilerillo"), una reconocida planta medicinal vulneraria (Martinez Crovetto 1980), y acompañarse con la toma de una infusión de Ribes magellanica Poir ("parrilla"), también de virtudes vulnerarias, anti-inflamatorias y analgésicas (Estomba et al. 2005; Meza \& Villagrán 1991).

La cantidad que habitualmente se prepara es de uno a dos litros, para lo cual se utilizan dos o tres ramas enteras, de aproximadamente $30-40 \mathrm{~cm}$ de longitud. Luego del hervor durante 10-15 minutos, el extracto se filtra y utiliza. El sobrante puede conservarse en botellas por varios días, para ser utilizado hasta una semana después de su preparación si se cuenta con un refrigerador en el hogar. Normalmente, este preparado se usa con la frecuencia en que cada persona suele higienizarse.

La recolección por otra parte, se realiza durante todo el año, aunque se considera que es preferible hacerla durante los meses de marzo y abril, "que es cuando las plantas se encuentran con la mayor fuerza, luego de haber pasado el verano y haber florecido" según los dichos de los pobladores. Las mujeres son en general, quienes la recolectan, preparan y administran.

En cuanto a las percepciones locales sobre el aroma de la planta en el campo, se registró que en general, es considerado suave y agradable (23\% de los registros). Esta información no parece ser relevante en su reconocimiento en la etapa de recolección, dado que las características morfológicas de la planta (principalmente su aspecto general y su color verde intenso) son las que determinan su localización. Por otro lado, en el proceso de preparación de la medicina, la intensidad del aroma que despide la planta al hervir, sumado al color que esta va adquiriendo, resultan de importancia como indicadores del estado de elaboración de la receta. Así, un aroma penetrante y un color verde-azulado marcan el punto preciso de la preparación.
Anatomía - Hoja.

Vista superficial (Fig. 1) - Las células epidérmicas propiamente dichas, son semejantes en ambas caras, poligonales en general, elongadas sobre los nervios, con paredes anticlinales engrosadas y con conspicuos campos de puntuaciones. Están recubiertas por una cutícula que presenta estriaciones características: radiales sobre las células que rodean a los tricomas, y concéntricas sobre las células vecinas a las células oclusivas de los estomas.

Los estomas son anomocíticos, rodeados por 4-6 generalmente 5 células subsidiarias. Están presentes en ambas superficies de la hoja. En la epidermis inferior presentan una longitud de $48,9 \mu \mathrm{m} \pm 4 \mu \mathrm{m}$ y un ancho de $48,5 \mu \mathrm{m} \pm 5 \mu \mathrm{m}$, mientras que en la epidermis superior presentan una longitud de $51,3 \mu \mathrm{m} \pm 6 \mu \mathrm{m}$ y un ancho de $47,79 \mu \mathrm{m} \pm 4 \mu \mathrm{m}$.

El indumento está formado por tricomas filiformes, 1-seriados, con un número de células que varía de 4 a 6 , las superiores de tipo glandular, en ambas epidermis. Se encuentran solitarios o reunidos en manojos.

Sección transversal (Fig. 2-5) - El espesor de la lámina a nivel de la costilla media es de $450 \mu \mathrm{m} \pm 50 \mu \mathrm{m}$.

Ambas epidermis son uniestratificadas, formadas por células cuadradas a rectangulares, con paredes anticlinales engrosadas y paredes periclinales internas algo más delgadas que las externas. La cutícula se presenta engrosada, en especial sobre la epidermis superior (Fig. 5). Los estomas se encuentran al mismo nivel o levemente por debajo que el resto de la epidermis, presentan paredes periclinales externas e internas engrosadas, lo que genera que el lumen tenga forma triangular. En las hojas más viejas, las paredes se encuentran más engrosadas, y el lumen se reduce adquiriendo forma de coma (Fig. 2 y 3 ).

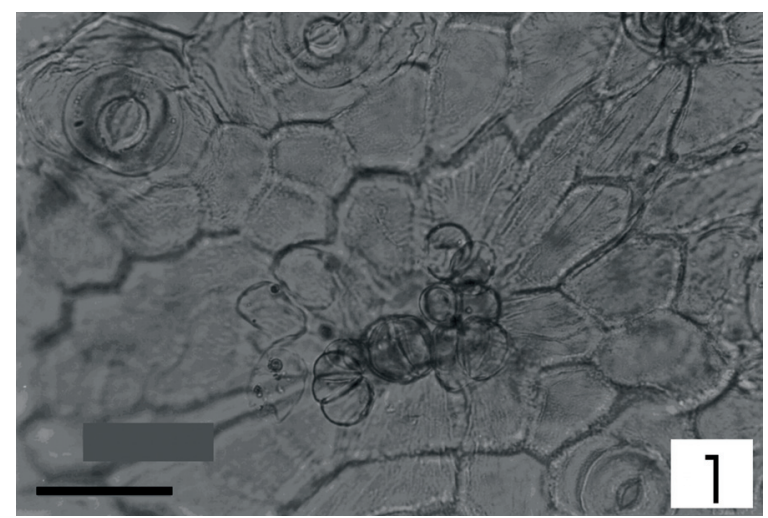

Figura 1. Vista superficial de la epidermis superior de la hoja de Baccharis obovata Hook. et Arn., exhibiendo tricomas glandulares en manojo, estomas anomocíticos y estriaciones de la cutícula. Reglilla $=$ $50 \mu \mathrm{m}$. 
La estructura del mesofilo es isobilateral, distinguiéndose un parénquima en empalizada superior formado por 3-4 hileras de células, un parénquima en empalizada inferior formado por 2-3 hileras, y un parénquima lagunoso central de 3-4 capas celulares (Fig. 2 y 3 ). Los haces vasculares son de tipo colateral, están rodeados por conspicuas vainas parenquimáticas, formadas por 1-2 capas de células con paredes engrosadas, a veces colenquimatosas. El haz vascular del nervio medio tiene contorno ovoide y normalmente se proyecta hacia la superficie inferior a modo de costilla
(Fig. 2). Se observa una depresión más o menos notable del epifilo sobre este nervio (Fig. 2 y 4). El colénquima está presente a nivel del nervio medio y une a modo de traba, el haz vascular central con las epidermis superior e inferior (Fig. 4). El esclerénquima se encuentra constituido por fibras agrupadas en casquetes sobre los polos de los haces (Fig. 2).

Cavidades secretoras se disponen asociadas a los haces vasculares, ubicadas entre el haz de fibras extrafloemático y las células de la vaina vascular, en número de 1-2 en la nervadura media (Fig. 2 y 4 ) y 1 en
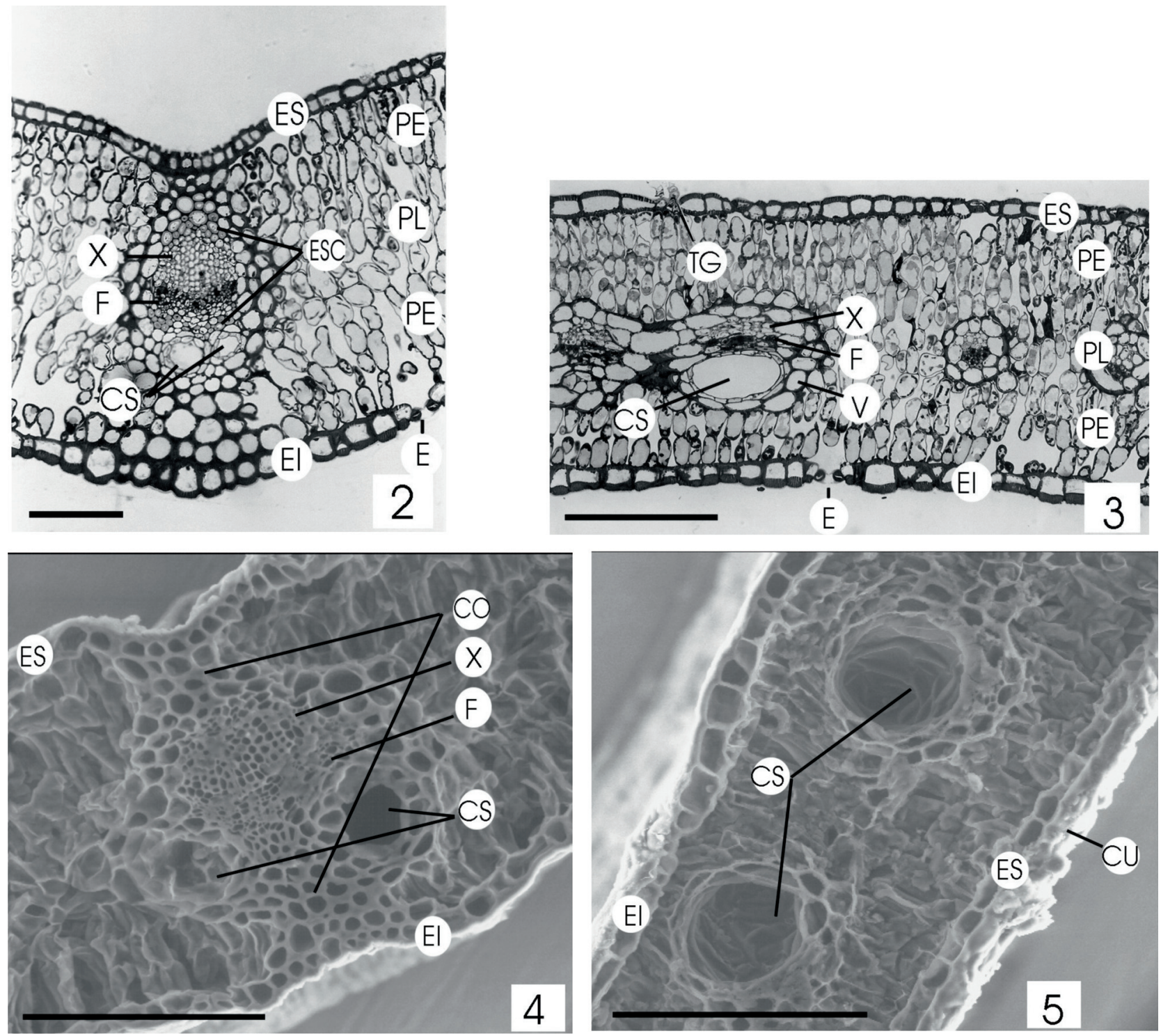

Figuras 2-5. Corte transversal de la hoja de Baccharis obovata Hook. et Arn. 2 y 3 (observación en microscopio óptico), 4 y 5 (en microscopio electrónico de barrido ambiental). 2 y 4 = aspecto general de la hoja al nivel del nervio central, mostrando la estructura isobilateral del mesofilo, el haz principal de tipo colateral, cavidades secretoras esquizógenas y estomas a nivel de la epidermis. 3 y $5=$ aspecto general de la hoja al nivel de los nervios secundarios, mostrando la estructura isobilateral del mesofilo y cavidades secretoras, tricomas glandulares (3) y la cutícula engrosada sobre epidermis superior (5). Reglillas en 2 y $3=60 \mu \mathrm{m}$, en 4 y $5=200 \mu \mathrm{m}$. Referencias: ES = epidermis superior; $\mathrm{PE}=$ parénquima en empalizada; $\mathrm{PL}=$ parénquima lagunoso; $\mathrm{EI}=$ epidermis inferior; $\mathrm{E}=$ estoma; $\mathrm{X}=\mathrm{xilema} ; \mathrm{F}=$ floema; $\mathrm{CS}=$ cavidad secretora; $\mathrm{TG}$ = tricoma glandular; $\mathrm{V}=$ vaina parenquimática; $\mathrm{CO}$ = colénquima; $\mathrm{CU}$ = cutícula; $\mathrm{ESC}$ = esclerénquima. 
las principales nervaduras laterales (Fig. 3 y 5 ). Presentan epitelio secretor entero formado por una, a veces dos capas de células. Las dimensiones de las cavidades asociadas al nervio medio son $39 \mu \mathrm{m} \pm 10 \times 47 \mu \mathrm{m} \pm 14$, y las de las asociadas a los nervios secundarios son de $24 \mu \mathrm{m} \pm 6 \times 31,5 \mu \mathrm{m} \pm 16$. Con el reactivo Sudán IV, el contenido se tiñe de naranja intenso.

Tallo (Fig. 6-8). El contorno es circular y ondulado, formado por valles y costillas más o menos prominentes (alrededor de 10-12 por corte).

La epidermis es uniestrata, compuesta por células rectangulares, y se encuentra cubierta por una gruesa cutícula (Fig. 7). Los estomas presentan paredes engrosadas, y se hallan al mismo nivel, o algo más elevados que el resto de la epidermis. Los tricomas son semejantes a los de las hojas, y se ubican principalmente en los valles.

En las costillas, se desarrolla tejido colenquimático. En los valles, puede presentarse un estrato de colénquima por debajo de la epidermis, o bien, varias capas de clorénquima, a menudo interrumpido por espacios de aire (Fig. 6 y 7).

El cilindro vascular está rodeado por una endodermis uniseriada, por sectores bisseriada (Fig. 6 y 7). Por debajo de esta, se disponen grupos de fibras esclerenquimáticas, en número de 10-13 por corte. Hacia adentro, el floema y el xilema forman cilindros contínuos. La médula es grande y está formada por células más o menos poligonales, de paredes gruesas, las más periféricas con cristales aciculares diminutos sueltos o en manojos (Fig. 8).

Cavidades secretoras ubicadas extraendodérmicamente, de $57,5 \mu \mathrm{m} \pm 8,5 \mu \mathrm{m} \times 59,3 \mu \mathrm{m} \pm$

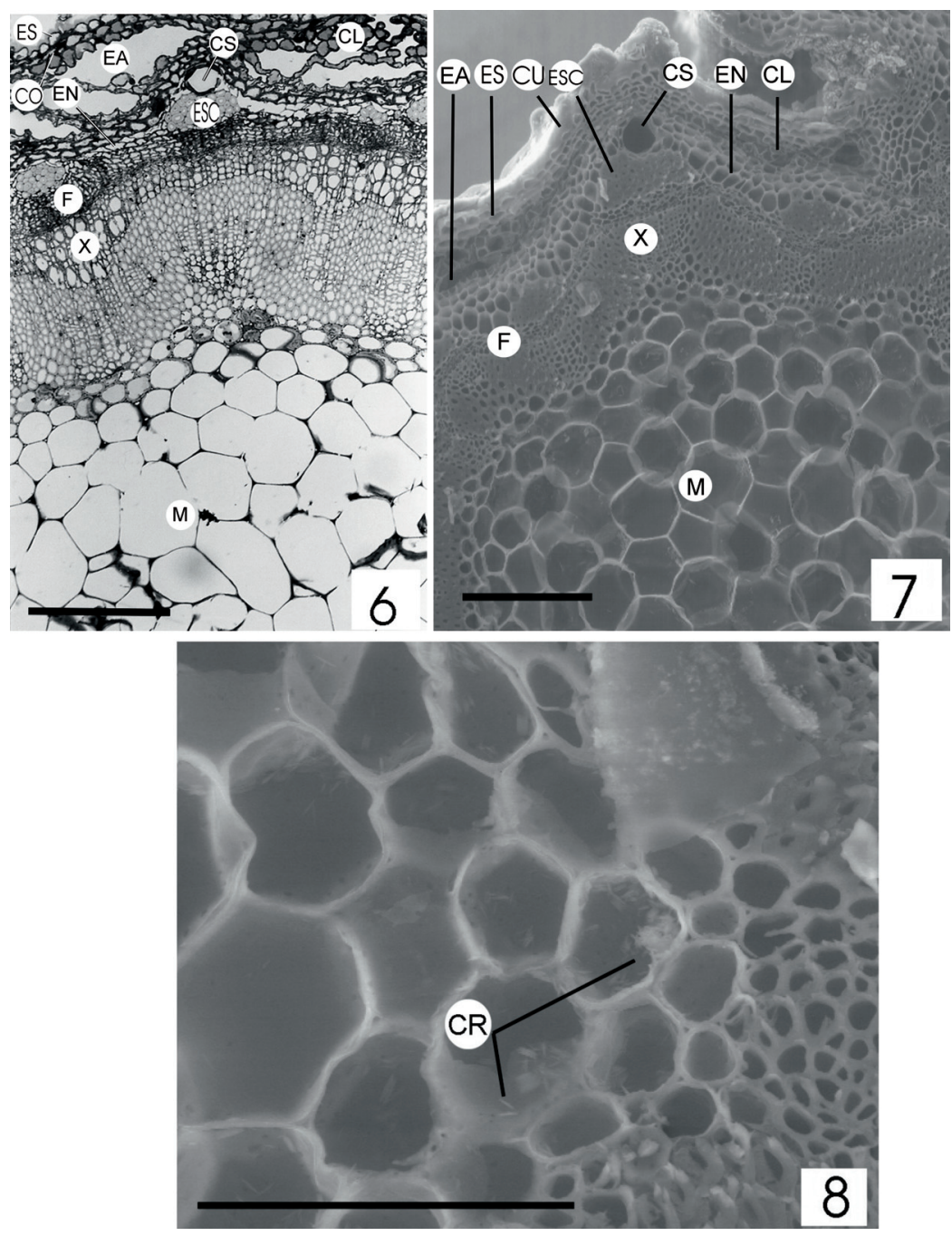

Figuras 6-8. Corte transversal del tallo joven de Baccharis obovata Hook. et Arn. 6 (observación en microscopio óptico), 7 y 8 (en microscopio electrónico de barrido ambiental). 6 y 7 = aspecto general del tallo, mostrando la médula, cilindro vascular, endodermis, cavidades secretoras extraendodérmicas, colénquima y esclerénquima alternados y la cutícula engrosada (7). $8=$ cristales aciculares en células de la periferia de la médula. Reglilla en 6 y $7=170 \mu \mathrm{m}$, en $8=100 \mu \mathrm{m}$. Referencias: ES = epidermis; $\mathrm{X}=$ xilema; F= floema; $\mathrm{CS}=$ cavidad secretora; $\mathrm{CO}=$ colénquima; $\mathrm{CU}=$ cutícula $\mathrm{EA}=$ espacio de aire $\mathrm{EN}=$ endodermis $\mathrm{ESC}=$ esclerénquima; $\mathrm{CL}=$ clorénquima; $\mathrm{M}=$ médula; $\mathrm{CR}=$ cristales. 
$16,4 \mu \mathrm{m}$, con epitelio secretor entero, se presentan enfrentadas a algunos de los grupos de fibras esclerenquimáticas (Fig. 6 y 7).

Fitoquímica - En la Tab. 1 se presentan los valores medios de las determinaciones físico-químicas realizadas, y las descripciones de las características organolépticas del aceite esencial en términos estandarizados.

Para el análisis del tiempo óptimo de destilación se agrega además, la curva de acumulación correspondiente (Fig. 9), donde puede observarse que el 59,8\% del total del aceite extraíble por hidrodestilación, se recoge en la primera hora del proceso, el 78,3\% durante la segunda hora, el 95,4\% en la tercer hora, y el 99,2\% del total en el transcurso de la cuarta hora. A partir de la quinta hora no se detecta que el proceso extractivo continúe separando aceite esencial.

Tabla 1. Valores medios de las determinaciones físico-químicas calculadas para el aceite esencial de Baccharis obovata Hook. et Arn.

\begin{tabular}{ll}
\hline Determinaciones físico-químicas & Valor medio \\
\hline Rendimiento medio anual & $2,57 \mathrm{~mL} / \mathrm{kg}$ peso oreado \\
& $4,03 \mathrm{~mL} / \mathrm{kg}$ peso seco \\
Tiempo óptimo de destilación & $5 \mathrm{horas}$ \\
Indice de refracción & $1,4800 \pm 0,0001$ \\
Densidad relativa & 0,884 \\
Rotación específica & $+12,4^{\circ} \pm 0,1^{\circ}$ \\
& $(0.2 ;$ ciclohexano) \\
Características organolépticas & Descripciones \\
Aroma & Nota inicial: especiosa, picante, \\
& algo cítrica \\
& Nota media: menos picante y \\
fresca & Nota de fondo: frutal, dulce \\
& Nota secundaria: resinosa \\
(luego de 8 horas)
\end{tabular}

\section{Discusión y conclusiones}

El presente trabajo aporta información actualizada e inédita sobre $B$. obovata, y verifica su mantenimiento en la actualidad como un recurso etnobotánico de importancia entre los habitantes de las comunidades Mapuches de Lago Rosario y Nahuelpan. Casi la mitad de los entrevistados todavía utiliza la planta, principalmente con fines cosmético-dermatológicos.

Se confirma que los órganos aéreos de la especie, son utilizados en el tratamiento de las cefalalgias y alopecia, tal como lo expusieran Martínez Crovetto (1980) y Kutschker et al. (2002). Se agregan datos sobre

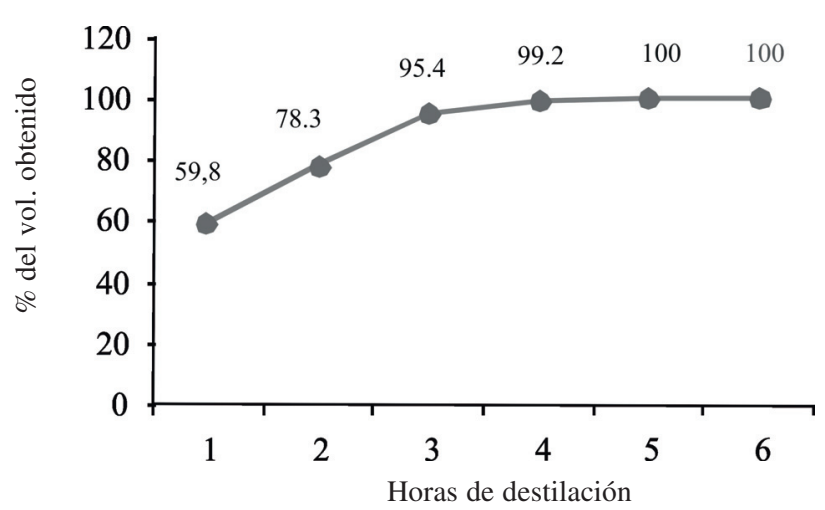

Figura 9. Curva de acumulación para la determinación del tiempo óptimo de destilación del aceite esencial de Baccharis obovata Hook. et Arn.

usos hasta el momento desconocidos, el detalle sobre los métodos de preparación y administración, de conservación y combinaciones con otras especies. Adicionalmente, se aporta nueva evidencia sobre el elevado consenso de uso de las especies aromáticas en la farmacopea Mapuche (p.e. Citarella 1995; Molares et al. 2007a; b; Molares \& Ladio 2008), información que resulta relevante al considerar el notable interés que vienen ganando las especies aromáticas en los últimos años, por su potencial comercial y medicinal (Bandoni 2003).

En cuanto a los principales usos señalados, se destacan tanto los cosméticos como los farmacológicos. Según Oumeish (2001), la cosmética ha sido una parte inseparable de la existencia diaria del ser humano, construyendo y modificando a lo largo de los siglos el concepto de belleza, mediante la búsqueda del mejoramiento de la apariencia, la corrección de los defectos y la decoración u ornamentación, incluyendo a la vez, la promoción de la protección y salud de las partes externas tratadas. Esta definición ha conducido al acuñamiento del concepto de "especie cosmecéutica" o "producto cosmecéutico", para hacer referencia a aquellas plantas cuyas aplicaciones son un híbrido entre las farmacéuticas y cosméticas (Millikan 2001). Este resulta particularmente adecuado para especies empleadas en el contexto de culturas tradicionales, donde las categorías medicinal y cosmética son a menudo artificiales, ya que ambas suelen ser percibidas como los polos de un continuo (Pieroni et al. 2004).

Por otro lado, hemos comprobado la vigencia del uso de B. obovata, lo cual resulta significativo considerando los procesos de cambio en los conceptos y prácticas de belleza y cosmética que frecuentemente se producen a través del tiempo en todas las sociedades, y que responden a fenómenos étnicos, económicos, históricos y/o políticos (Oumeish 2001). Este hecho podría responder a distintos factores, entre ellos; los 
586 Molares, González, Ladio \& Castro: Etnobotánica, anatomía y caracterización físico-química del aceite esencial...

económicos, pues acceder a los productos del mercado es limitante para la mayoría de los hogares; los prácticos, relacionados a la buena disponibilidad de la especie en la región; a factores culturales, como la tradición de uso; y a la eficacia empíricamente demostrada que se indica.

En relación a la indagación organoléptica, encontramos que para los entrevistados el aroma de la planta no constituye un carácter contrastante para su reconocimiento en el campo. Esto podría estar vinculado a la intensidad del mismo, que considerado suave, resulta dificultoso percibirlo. En términos especulativos, esto se relacionaría en parte, a una baja concentración de las moléculas odoríferas en el aire (Amat \& Vincent 2003), lo que entre otros motivos, sería una consecuencia de la anatomía particular de la planta, que "encierra" en cavidades secretoras los productos aromáticos, aislándolos del medio externo (Bandoni 2003). De otra manera, el aroma que esta emite al entrar en ebullición sí les aporta información significativa sobre el mejor modo de prepararla y administrarla, tal como sucede en muchas sociedades tradicionales (Schmeda-Hirschmann \& Yesilada 2005). Según Schmeda-Hirschmann \& Yesilada (2005) este tipo de información complementaria, suele estar ausente en los trabajos etnofarmacológicos, aunque resulta indispensable en la validación científica, dado que reduciría el tiempo de los ensayos que prueban las concentraciones óptimas y otros parámetros farmacológicos.

Por su parte, las evaluaciones organolépticas realizadas en laboratorio, agregan información complementaria a las realizadas en el campo, al sumar evidencia para la caracterización del aceite esencial. Cabe señalar, que las apreciaciones organolépticas sobre el vegetal y sobre el extracto, no son comparables netamente entre sí, por haber surgido de la evaluación de materiales diferentes. Por ejemplo, en una planta viva no pueden distinguirse los aromas correspondientes a los aceites esenciales de los de otros productos aromáticos. Tampoco es posible diferenciar los componentes más pesados de los más livianos y volátiles del aceite, ya que los segundos enmascaran continuamente a los primeros, cosa que no ocurre si se huele el aceite puro en laboratorio, donde a medida que se van evaporando las fracciones más livianas, se van detectando los componentes más pesados (Bandoni 2003). Además, ambas apreciaciones responden a las percepciones propias de dos sociedades distintas (Classen 2002). Sin embargo, es sabido que la quimio-percepción del aroma es primeramente un proceso biológico vinculado a la detección química de las sustancias odoríferas, posible a través del olfato, el gusto y el sistema nervioso trigeminal; que luego es interpretado y valorado en términos culturales (Brett \& Heinrich 1998). En nuestro estudio encontramos que entre las apreciaciones de los entrevistados sobre el aroma de la preparación y nuestra propia apreciación sobre el aroma del aceite esencial, se comparte la idea de intensidad. En este punto lo que nosotros pudimos separar de forma artificial en notas "picantes" y fuerza "moderadamente fuerte", los informantes lo sintetizaron en aroma "penetrante". El aroma que se desprende de la preparación correspondería a diversas sustancias volátiles, entre ellas los aceites esenciales estudiados. Estos aceites al evaporarse abandonan la preparación, y se detectan y evalúan a través de los mecanismos señalados anteriormente. De esta forma se encuentra que los informantes pueden distinguir diferentes conjuntos de sustancias químicas durante el proceso de ebullición, en este caso componentes volátiles de los aceites esenciales, lo cual lo aprovechan como indicador del punto justo de la preparación.

En cuanto a las variables físico-químicas estudiadas, encontramos que el rendimiento medio anual calculado $(4,03 \mathrm{~mL} / \mathrm{kg}$ peso seco) presenta un valor intermedio en comparación con el de otras especies del género, como por ejemplo B. gaudichaudiana DC (1mL/kg) (Budel et al. 2004), B. myrtilloides Griseb. (Syn.: B. tucumanensis Hook. et Arn.) $(2 \mathrm{~mL} / \mathrm{kg})$, B. rufescens Spreng. (2 mL/kg) (Zunino et al. 1998), B. salicifolia (Ruiz \& Pav.) Pers. (1,6-3 mL/kg) (Loayza et al. 1995), B. trimera (Less.) DC. (3 mL/kg) (Siqueira et al. 1986), B. latifolia (Ruiz \& Pav.) Pers. (1,8-4,5 mL/kg) (Loayza et al. 1995), B. articulata (Lam.) Pers. ( $5 \mathrm{~mL} / \mathrm{kg}$ ), B. cylindrica (Syn.: B. crispa Spreng.) $(6 \mathrm{~mL} / \mathrm{kg})$, B. dracunculifolia DC ( $8 \mathrm{~mL} / \mathrm{kg})$ (Budel et al. 2004). Así mismo, según la categorización propuesta por el organismo de Ciencia y Tecnología para el Desarrollo (CYTED), el valor de rendimiento de B. obovata es bajo a intermedio, dado que valores menores a $5 \mathrm{~mL} / \mathrm{kg}$ los considera bajos, valores entre $5 \mathrm{~mL} / \mathrm{kg}$ y $10 \mathrm{~mL} / \mathrm{kg}$ intermedios, y valores superiores a $10 \mathrm{~mL} / \mathrm{kg}$ altos. Esta información es particularmente utilizada como un criterio más para la selección de especies aromáticas potencialmente comercializables. Sin embargo, la misma debe analizarse en conjunto con otras características del aceite esencial, como la originalidad del aroma, composición química y efectos farmacológicos (Bandoni 2003). En el caso del aceite esencial de B. obovata, es de destacar su fuerte, persistente y distintivo aroma, lo que podría considerarse valioso en este sentido. En relación a lo dicho, pueden citarse numerosos ejemplos de especies con rendimientos relativamente bajos pero altamente reconocidas y explotadas por sus cualidades medicinales, cosméticas y/o condimenticias, como Achillea millefolium L. (Georgia et al. 2005), Aloysia citriodora Palau (Abella 1998), Tagetes minuta L. (Abella 
1998) y Mentha spicata L. (Bandoni 2003), entre otras.

En relación a las características de color y aspecto del aceite (levemente amarillo, uniforme y translúcido), se concluye que son las normales para la mayoría de los aceites esenciales conocidos. Cualquier cambio evidenciable sobre estas, indicaría una posible descomposición u adulteración del mismo (Bandoni 2003). La densidad estimada $(0,884)$ por su parte, es menor a la del agua, por lo que este aceite se clasifica como "ligero" (Bandoni 2003). Respecto al tiempo óptimo de destilación establecido (5 horas) podrá utilizarse en una eventual explotación del aceite esencial, para maximizar los tiempos de producción y homogeneizar la calidad del mismo.

La investigación anatómica por su parte, permite indicar que los tricomas glandulares del tipo presentes en $B$. obovata también se encuentran en otras especies del género, por ejemplo en $B$. articulata (Lam.) Pers. (Ariza Espinar 1969; Cortadi et al. 1999), B. grisebachii Hieron. (Ariza Espinar 1969), B. gaudichaudiana DC. (Budel et al. 2003), B. sagittalis (Less.) DC., B. triangularis Hauman (Petenatti et al. 2007) y B. linearis (Ruiz et Pav.) Pers. (Montenegro 1981). Según Montenegro (1981) estos podrían contener metabolitos secundarios capaces de reprimir el ataque de ciertos herbívoros. Los estomas de tipo anomocíticos son mencionados para la mayor parte de las especies del género (Ariza Espinar 1969; Cortadi et al. 1999; Budel et al. 2003; Budel \& Duarte 2007; Petenatti et al. 2007), aunque algunas también presentan el tipo anisocítico, como B. articulata (Lam.) Pers y B. crispa Spreng. (Ariza Espinar 1969; Cortadi et al. 1999), ausentes en B. obovata. La cutícula gruesa y estriada también se ha encontrado en otras especies del género (Cortadi et al. 1999; Budel et al. 2003; Budel \& Duarte 2007; Petenatti et al. 2007). Las cavidades secretoras de las hojas se encuentran asociadas a los haces vasculares, rodeadas por la vaina parenquimática, conforme también a lo usualmente observado en el género (Ariza Espinar 1969).

El tallo presenta clorénquima y colénquima alternados, y cilindro vascular organizado también de forma similar a lo observado en otras especies medicinales del género (Ariza Espinar 1969; Cortadi et al. 1999; Budel et al. 2003; Budel \& Duarte 2007; Petenatti et al. 2007). La endodermis presente en B. obovata es frecuente en el tallo y la raíz de las Asteraceae (Ariza Espinar 1969) y delimita internamente el cilindro vascular de la corteza. En las proximidades de esta se observan cavidades secretoras esquizógenas extraendodérmicas semejantes a las encontradas en $B$. pingraea DC. (Ariza Espinar 1969), B. crispa Spreng. y B. articulata (Lam.) Pers. (Ariza Espinar 1969; Cortadi et al. 1999; Budel et al. 2003). Las células periféricas del parénquima medular contienen cristales, lo cual es frecuente en el género (Ariza Espinar 1969; Cortadi et al. 1999; Budel et al. 2003; Budel \& Duarte 2007; Petenatti et al. 2007).

Por otro lado, la combinación de los caracteres anatómicos encontrados indicaría una estrategia adaptativa tendiente a la economía de agua. En este sentido, las características más sobresalientes son: la epidermis con paredes gruesas y una cutícula engrosada, que proporcionarían rigidez a la lámina frente a los fuertes vientos, evitando a la vez, el quiebre de la misma por marchitamiento durante la estación seca (Ragonese 1990; Montenegro et al. 1981); el mesofilo de estructura isobilateral, compacto, con un parénquima en empalizada desarrollado, el que aumentaría la resistencia a la pérdida de agua (Ragonese 1990); y estructuras secretoras en el mesofilo, responsables de la secreción de aceites esenciales, como estructuras frecuentes en plantas que crecen en regiones con climas adversos, en particular donde el período seco coincide con el período de temperaturas más elevadas (Langenheim 1994), como es el caso de la Patagonia.

Dado que la estructura anatómica general de $B$. obovata se asemeja a la de otras especies del género, se hace imprescindible considerar los parámetros cuantitativos que se aportan (dimensiones de estomas, cavidades secretoras, tricomas, etc.) y las características morfológicos de la especie, ya descriptas en otro trabajo (Cabrera 1971), de forma conjunta, a los fines de diferenciar y distinguir correctamente esta especie del resto. Así mismo es de resaltar, que la descripción anatómica y ubicación de las estructuras secretoras, cobra valor para la selección del mejor método de extracción de los aceites esenciales (Duke 1994; WHO 1998), en miras a un potencial aprovechamiento comercial del mismo.

Finalmente, creemos que este trabajo puede ser de utilidad a futuras tareas de validación y control de calidad, tanto de la droga cruda como de sus extractos. A la vez, pensamos que puede ser un aporte al desarrollo de un enfoque integrador de diferentes disciplinas, en este caso la anatomía, fitoquímica y etnobotánica, que contribuya a la comprensión más acabada de los factores biológicos y culturales que intervienen en los procesos de selección y uso de las especies medicinales utilizadas por los pueblos aborígenes (Gonzalez-Tejero \& Casares-Porcel 1996; Rodríguez 2000; Molares et al. 2007 a). También, consideramos que será indispensable realizar estudios farmacológicos y médicos que corroboren la eficacia y dosis de toxicidad del extracto acuoso, entre otras investigaciones pertinentes, que permitan en el fututo una promoción oficial de sus virtudes medicinales y tal vez, su posible incorporación a las prácticas de atención primaria de la salud. 
588 Molares, González, Ladio \& Castro: Etnobotánica, anatomía y caracterización físico-química del aceite esencial...

\section{Agradecimientos}

Un especial agradecimiento a los pobladores de Lago Rosario y Nahuelpan, quienes generosamente brindaron su saber y amistad durante los meses de trabajo en el campo. Al Consejo Nacional de Investigaciones Científicas y Técnicas, a la Universidad Nacional del Comahue y Universidad Nacional de la Patagonia San Juan Bosco, por la financiación parcial de la investigación. Al Ing. Pedro Guerra y a la Dra. Beatriz Varela por su asesoramiento en técnicas anatómicas, y a la Ing. Magdalena Aguirre por su colaboración en las lecturas físico-químicas.

\section{Referencias bibliográficas}

Abad, M.J. \& Bermejo, P. 2007. Baccharis (Compositae): A review update. ARKIVOC (vii): 76-96.

Abella, L. 1998. Flora medicinal andina: Plantas aromáticas usadas en la terapéutica popular. En: Tesis Doctoral. Facultad de Ciencias Naturales y Museo, Universidad Nacional de La Plata, Argentina.

Alexiades, M.N. 1996. Selected guidelines for ethnobotanical reserach: A field manual. New York, The New York Botanical Garden.

Amat, J.M. \& Vincent, J.D. 2003. Una nueva fisiología del gusto. Barcelona, RBA Libros.

Ariza Espinar, L. 1969. Las especies de Baccharis (Compositae) de Argentina Central. Tesis Doctoral. Buenos Aires, Universidad Nacional de Bs. As., Fac. de Farmacia y Bioquímica.

Bandoni, A. 2003. Los recursos vegetales aromáticos en Latinoamérica. Su aprovechamiento industrial para la producción de aromas y sabores. Buenos Aires, Ed. de la U.N.L.P.

Brett, A.J. \& Heinrich, M. 1998. Culture, perception and the environment: the role of chemosensory. Angewandte Botanik 72, 67-69.

Budel, J.M.; Duarte, M. \& Santos, C.A. 2003. Caracteres morfoanatômicos de Baccharis gaudichaudiana DC., Asteraceae. Acta Farmacéutica Bonaerense 22: 313-20.

Budel, J.M.; Duarte, M.R. \& Santos, C.A.M. 2004. Parâmetros para análise de carqueja: Comparação entre quatro espécies de Baccharis spp. (Asteraceae). Revista Brasileira de Farmacognosia 14: 41-48.

Budel, J.M. \& Duarte, M.R. 2007. Caracteres morfoanatômicos de partes vegetativas aéreas de Baccharis coridifolia DC. (AsteraceaeAstereae). Latin American Journal of Pharmacy 26: 723-731.

Cabrera, A.L. 1971. Compositae. Parte VII. Tomo VIII. In: M. N Correa (ed.). Flora Patagónica. Buenos Aires, Colección científica del INTA.

Catalán Labarías, R. \& Ramos Antiqueo, R. 2008. Los bosques nativos del sur de Chile y el pueblo mapuche. World Rainforest Movement. Additional information. Disponible en: http:// www.wrm.org.uy/plantations/information/Catalan2.html (Acceso: mayo 2008).

Chaudhury, R.R. 1992. Herbal medicine for human health. Regional Publication, SEARO, NÚ 20. World Health Organization. New Dehi, Regional Office for South-East Asia.

Citarella, L. 1995. Medicinas y culturas en la Araucanía. Chile, Ed. Sudamericana. Santiago.

Classen, C. 2002. Fundamentos de una antropología de los sentidos. Disponible en: http://www.unesco.org/issj/rics153/ classenspa.html (Acceso: mayo 2007).
Cobos, M.I.; Rodriguez, J.L.; Oliva, M.M.; Demo, M.; Faillaci, S.M. \& Zygadlo, J.A. 2001. Composition and antimicrobial activity of the essential oil of Baccharis notosergila Griseb. Planta Medica 67: 84-86.

Conticello, L.; Gandullo, R.; Bustamante, A. \& Tartaglia, C. 1997. El uso de plantas medicinales por la comunidad Mapuche de San Martín de los Andes, provincia de Neuquén (Argentina). Parodiana 10: 165-180.

Correa, M.N. 1998. Flora patagónica. Parte I. Buenos Aires, Colección Científica del INTA.

Cortadi, A.; Sapio, O.D.; Cargo, J.Mc.; Scandizzi, A.; Gattuso, S. \& Gattuso, M. 1999. Anatomical studies of Baccharis crispa and Baccharis trimera, "Carquejas" used in folk medicine. Pharmaceutical Biology 37: 357-365.

Cutter, E.G. 1978. Plant anatomy. Part I. Cells and tissues. London, Arnold.

CYTED, subprograma IV. 20: Normalización de productos naturales obtenidos de especies de la flora aromática Latinoamericana. Disponible en: http://www.cyted.org (Acceso: 2003).

D’Ambrogio de Argueso, A. 1986. Manual de técnicas en histología vegetal. Buenos Aires, Ed. Hemisferio Sur.

Dimitri, M.J. 1977. Pequeña flora ilustrada de los Parques Nacionales Andino-Patagónicos. Publicación técnica $\mathrm{N}^{\circ}$ 46. $2^{\text {da }}$ ed. Buenos Aires, Servicio Nacional de Parques Nacionales.

Duke, S.O. 1994. Commentary: Glandular trichomes- A focal point of chemical and structural interactions. International Journal of Plant Sciences 155: 617-620.

Estomba, D.; Ladio, A. \& Lozada, M. 2005. Plantas medicinales utilizadas por una comunidad Mapuche en las cercanías de Junín de los Andes, Neuquén. Boletín Latinoamericano y del Caribe de Plantas Medicinales y Aromáticas 6: 107-112.

Estomba, D.; Ladio, A. \& Lozada, M. 2006. Medicinal wild plant knowledge and gathering patterns in a Mapuche community from North-western Patagonia. Journal of Ethnopharmacology 103: 109-119.

Feresin, G.E.; Tapia, A.; Gimenez, A.; Gutierrez Ravelo, A.; Zacchino, S.; Sortino, M. \& Schmeda-Hirschmann, G. 2003. Constituents of the Argentinian medicinal plant Baccharis grisebachii and their antimicrobial activity. Journal of Ethnopharmacology 89: $73-80$.

Fahn, A. 1979. Secretory tissues in plants. London, New York, San Francisco, Academic Press.

Farmacopea Europea. 1988. Vol. 4.5.8. $2^{\text {da }}$ ed.

Giorgia A.; Bononi, M.; Tateo, F. \& Cocucci, M. 2005. Yarrow (Achillea millefolium L.) growth at different altitudes in central Italian alps: Biomass yield, oil content and quality. Journal of herbs, spices \& medicinal plants 11: 47-58.

Giuliano, D.A. \& Ariza Espinar, L. 1999. Asteraceae: Baccharis Pp: 113-129. In: F.O. Zuloaga \& O. Morrone (eds.). Catálogo de las plantas vasculares de la República Argentina II. Missouri Botanical Garden N 74.

González, S.B. \& Molares, S. 2004. Plantas medicinales utilizadas en comunidades rurales del Chubut, Patagonia Argentina. Boletín Latinoamericano y del Caribe de Plantas Medicinales y Aromáticas 3: 58-62.

González-Tejero, M.R. \& Casares-Porcel, M. 1996. La anatomía vegetal como método de identificación en etnobotánica. Monografías del Jardín Botánico de Córdoba 3: 33-37.

Instituto Nacional De Estadística y Censos. 2001. Censo nacional de población, hogares y viviendas. Argentina.

IRAM- SAIPA N ${ }^{\circ}$ 185-04. 1981. Método de la determinación de la densidad relativa. CDU 668.5: 531. 755.1.

IRAM- SAIPA N ${ }^{\circ}$ 185-05. 1982. Método de determinación del índice de refracción. CDU 668. 5: 535. 324.

IRAM- SAIPA No 185-07. 1987. Método de determinación de rotación óptica. CDU 668. 5: 541. 653.

IRAM- SAIPA No 186-01. 1970. Método de valoración olfativa 
CDU 668.5:620.1.

Kutschker, A.; Menoyo, H. \& Hechem, V. 2002. Plantas medicinales de uso popular en comunidades del oeste del Chubut. Bariloche, INTA Esquel - Universidad Nacional de la Patagonia S.J.B. - GTZ.

Ladio, A.H. 2006. Gathering of wild plant foods with medicinal use in a Mapuche community of northwest Patagonia. Pp: 297-321. Chapter 13. In: A. Pieroni \& L.L. Price (eds.). Eating and healing. Traditional foods as medicine. Food Products Press.

Langenheim, J.H. 1994. Higher plant terpenoids: a phytocentric overview of their ecological roles. Journal of Chemical Ecology 20: 1223-1280.

Leonti, M.; Sticher, O. \& Heinrich, M. 2002. Medicinal plants of the Popoluca, México: Organoleptic properties as indigenous selection criteria. Journal of Ethnopharmacology 81: 307-315.

Loayza, I.; Abujder, D.; Aranda, R.; Jakupovic, J.; Collin, G.; Deslauriers, H. \& Jean, F. 1995. Essential oils of Baccharis salicifolia, B. latifolia and B. dracunculifolia. Phytochemistry 38: 381-389.

Malizia, R.A.; Cardell, D.A.; Molli, J.S.; González, S.B.; Guerra, P.E. \& Grau, R.J. 2005 a. Volatile constituents of leaf oils from the genus Baccharis. Part I. B. racemosa (Ruiz et. Pav.) De Candolle and $B$. linearis (Ruiz et. Pav.) Persoon species from Argentina. Journal of Essential Oil Research 17: 103-106.

Malizia, R.A.; Cardell, D.A.; Molli, J.S.; González, S.B.; Guerra, P.E. \& Grau, R.J. 2005b. Volatile constituents of leaf oils from the genus Baccharis. Part II. B. obovata Hooker et Arnott and B. salicifolia (Ruiz et. Pav.) Persoon species from Argentina. Journal of Essential Oil Research 17: 194-197.

Martínez Crovetto, R. 1980. Apuntes sobre la vegetación de los alrededores del Lago Cholila. Publicación Técnica de la Facultad de Ciencias Agrarias 1: 1-22.

Messer, E. 1991. Systematic and medicinal reasoning in Mitla folk botany. Journal of Ethnopharmacology 33: 107-128.

Meza, P. \& Villagrán, C. 1991. Etnobotánica de la isla de Alao, archipiélago de Chiloe, Chile. Boletín del Museo Nacional de Historia Natural 42: 39-78.

Millikan, L.E. 2001. Cosmetology, cosmetics, cosmeceuticals: definitions and regulations. Clinics in Dermatology 19: 371-374

Molares, S.; Ladio, A.H. \& Vobis, G. 2007 a. Etnobotánica y anatomía foliar de las especies de Chiliotrichum Cass. (Asteraceae: Astereae): Ch. rosmarinifolium Less. y Ch. diffusum (Forst.) O. Kuntze. Kurtziana 33: 5-16.

Molares, S.; Ladio, A. \& Castro, M.A. 2007 b. Etnobotánica, percepciones organolépticas y estucturas secretoras de cinco especies aromáticas de la Patagonia Argentina. Boletín Latinoamericano y del Caribe de Plantas Medicinales y Aromáticas 6: 209-210.

Molares, S. \& Ladio, A. 2008. Plantas medicinales en una comunidad Mapuche del NO de la Patagonia Argentina: Clasificación y percepciones organolépticas relacionadas con su valoración.
Boletín Latinoamericano y del Caribe de Plantas Medicinales y Aromáticas 7: 149-155.

Montenegro, G.; Segura, B.; Saenger, R. \& Mujica, A.M. 1981. Xeromorfismo en especies arbustivas del matorral chileno. Anales del Museo de Historia Natural 14: 71-83.

Oumeish, Y.O. 2001. The cultural and philosophical concepts of cosmetics in beautyand art through the medical history of mankind. Clinics in Dermatology 19: 375-386.

Petenatti, E.M.; Petenatti, M.E.; Cifuente, D.A.; Gianello, J.C.; Giordano, O.S.; Tonn, C.E. \& Del Vitto, L.A. 2007. Medicamentos herbarios en el centro-oeste Argentino. VI. Caracterización y control de calidad de dos especies de "Carquejas": Baccharis sagittalis y $B$. triangularis (Asteraceae). Latin American Journal of Pharmacy 26: 201-208.

Phillips, O. 1993. The potential for harvesting fruits in tropical rainforests: new data from Amazonian Peru. Biodiversity and Conservation 2: 18-38.

Pieroni, A.; Quave, C.L.; Villanelli, M.L.; Mangino, P.; Sabbatini, G.; Santini, L.; Boccetti, T.; Profili, M.; Ciccioli, T.; Rampa, L.G.; Antonini, G.; Girolamini, C.; Cecchi, M. \& Tomasi, M. 2004. Ethnopharmacognostic survey on the natural ingredients used in folk cosmetics, cosmeceuticals and remedies for healing skin diseases in the inland Marches, Central-Eastern Italy. Journal of Ethnopharmacology 91: 331-344.

Ragonese, A.M. 1990. Caracteres xeromorfos foliares de Nassauvia lagascae (Compositae). Darwiniana 30: 1-10.

Rodríguez, M.F. 2000. Woody plant species used during the archaic period in the Southern Argentine Puna. Archaeobotany of Quebrada Seca 3. Journal of Archaeological Science 27: 341-361.

Schmeda-Hirschmann, G. \& Yesilada, E. 2005. Traditional medicine and gastroprotective crude drugs. Journal of Ethnopharmacology 100: 61-66.

Siqueira, N.C.S.; Silva, G.A.A.B. \& Alice, C.B. 1986. Análise dos óleos essenciais de algumas plantas aromáticas tradicionais ou nativas no Rio Grande do Sul. Revista Brasileira de Farmácia 67: $118-128$.

WHO. 1998. Quality control methods for medicinal plant materials. Geneva, WHO Library.

Zunino, M.P.; Newton, M.N; Maestri, D.M. \& Zygadlo, J.A. 1997. Composition of the essential oil of Baccharis crispa Spreng. and Baccharis salicifolia Pers. grown in Córdoba (Argentina). Flavour and Fragrance Journal 12: 405-407.

Zunino, M.P.; Newton, M.N.; Maestri D.M. \& Zygadlo, J.A. 1998. Essential oils of three Baccharis species. Planta Medica 64: 86-87.

Zunino, M.P.; Lopez, M.L.; Faillaci, S.M.; Lopez, A.G.; Ariza Espinar, L. \& Zygadlo, J.A. 2000. Essential oil of Baccharis cordobensis Heering. Flavour and Fragrance Journal 15: 151-152.

Zunino, M.P.; Lopez, M.L.; Zygadlo, J.A. \& Lopez, A.G. 2004. Essential oil composition of Baccharis articulata (Lam.) Pers. J. Essential Oil Research 16: 29-30. 